\title{
THE CHOICE OF CELL SIZE AND THE USE OF PRE-CALCULATED CORRECTION FACTORS IN THE ANALYSIS OF PLANAR CIRCUITS USING FDTD AND TLM.
}

\author{
Chris J Railton \\ Centre for Communications Research, Faculty of Engineering \\ University of Bristol, Bristol, England, BS8 1BU \\ Phone: (0)1179545175 Fax: (0)1179545206 Email chris.railton@ bristol.ac.uk
}

\begin{abstract}
In this contribution it is shown that small changes to the choice of cell size in the FDTD and TLM methods can have a large effect on the predicted behaviour of a microstrip filter but that the use of Pre-calculated Correction Factors $(P C F s)$ can vastly reduce this effect and also yield more accurate answers for a specified cell size.
\end{abstract}

\section{INTRODUCTION}

The Finite Difference Time Domain (FDTD) and Transmission Line Matrix (TLM) methods have both been much used for the analysis of microstrip circuits, such as filters. However, because (i) the width of the strips in such circuits are normally a small fraction of the wavelength, (ii) the behaviour of a filter is very sensitive to exact width of the strips and (iii) the fields vary rapidly near metal edges, much care must be taken. In this contribution it is shown that small changes to the choice of cell size can have a large effect on the predicted behaviour of a microstrip filter but that the use of Precalculated Correction Factors (PCFs) can vastly reduce this effect and also yield more accurate answers for a specified cell size.

As an example, consider the low pass filter shown in Figure 1. All the microstrip tracks, apart from the two feed lines, have a width of $0.26 \mathrm{~mm}$ and therefore present a challenge to the FDTD or TLM modeller who wishes to avoid the use of an excessively fine mesh with consequent large amount of computer time. A cell size which would be expected to give a good performance in regard to dispersion would be $\lambda_{\mathrm{g}} / 20$ which for frequencies up to $6 \mathrm{GHz}$ is approximately $1.5 \mathrm{~mm}$. However, in the basic method the narrow microstrips dictate a cell size much smaller than this for reliable results. The aim of this research is to enhance the basic methods to allow a larger cell size to be used without sacrificing accuracy.

\section{THE EFFECT OF CELL SIZE AND POSITION}

For a cell size of $0.26 \mathrm{~mm}$ the staircase approximation will, depending on the position of the strip relative to the edge of the cell, force the strip to be modelled as having a zero nominal width or as having the width of one cell. Fringing effects will make the track look as if it is actually wider than these nominal widths by approximately $40 \%$ of the cell size ( $20 \%$ for each edge ). In the case of the $0.5 \mathrm{~mm}$ mesh, the strip will always be approximated as being infinitesimal. In Figure 2 the predicted results with these three choices of mesh are shown. It can be seen that there is a large discrepancy between the two instances of a $0.26 \mathrm{~mm}$ mesh being used. It can also be seen that the results using the coarser $0.5 \mathrm{~mm}$ mesh are little different from those obtained using the $0.26 \mathrm{~mm}$ mesh with the infinitesimal width approximation.

Corresponding results obtained using the TLM method with Super Symmetrical Condensed Nodes (SSCN) are shown in Figure 3. Here it can be seen that the choice of mesh size and position has a very similar effect on results as for the FDTD method.

It is noted that in FDTD the strip edges are usually aligned so that they coincide with the longitudinal E field nodes. Thus, the edges pass through the origin of the FDTD cell. For TLM SSCN, the strip edges must be pass through the centre of the face of the TLM cell. Thus, for a strip on the xz plane and orientated in the $\mathrm{z}$ direction, the edges must be offset from the origin of the cell by half a cell size in the $\mathrm{x}$ direction. Similarly, for the strips orientated in the $\mathrm{x}$ direction the edges must be offset by half a cell size in the $\mathrm{z}$ direction. This is particularly important when the width of the strips is approximated to being infinitesimal. In order to make the comparison between the methods as close as possible, the 
whole structure was shifted by half a cell size in each of the directions tangential to the plane of the filter when changing from FDTD to TLM.

Another example of the effect of altering the FDTD cell sizes, this time with a non-uniform mesh, is shown in Figure 4 Figure 6. In this example a fine mesh is used in the vicinity of the narrow tracks in order to more accurately model them. In this filter it is this part of the structure which affects the position of the first resonance. In mesh " $\mathrm{f}$ ", the cell size at the $\mathrm{x}$ directed microstrip tracks is reduced from $0.5 \mathrm{~mm}$ to $0.13 \mathrm{~mm}$ so that the track is exactly two cells wide. In mesh " $\mathrm{h}$ ", a block of narrow cells is placed around the $\mathrm{x}$ directed microstrip tracks. The " $\mathrm{g}$ " mesh is a uniform $0.5 \mathrm{~mm}$ mesh. The predicted results from each of these meshes is shown in Figure 6. It can be seen that, perhaps surprisingly, adding the bands of narrow cells makes almost no difference to the position of the first resonance. On the other hand, using just two narrow cells at each track causes a small but definite increase in resonant frequency. This can be explained as follows: For the " $\mathrm{g}$ " mesh in which the track is nominally infinitesimal but the cell size is $0.5 \mathrm{~mm}$, the actual modelled width will be approximately $0.2 \mathrm{~mm}$. For the "f" mesh where the nominal width is $0.26 \mathrm{~mm}$ but the size of the cells next to the edges are $0.5 \mathrm{~mm}$, the modelled width will be approximately $0.46 \mathrm{~mm}$. Thus the inductance of the track appears less and the predicted resonant frequency is higher. For the " $\mathrm{h}$ " mesh the nominal width is $0.26 \mathrm{~mm}$ and the surrounding cells are $0.13 \mathrm{~mm}$ so the modelled width is approximately $0.27 \mathrm{~mm}$ which is only slightly wider than for the uniform coarse mesh.

In fact, it turns out that, fortuitously, the results using the " $\mathrm{f}$ " mesh are more accurate than the others even though the strip widths are being less accurately represented. This is because other parts of the structure are also being imperfectly approximated and the errors are tending to cancel. This emphasises the importance of ensuring that all edges are correctly accounted for and the ease of being misled into believing that a model is sound because the correct result is obtained in one instance.

\section{THE EFFECT OF USING PRE-CALCULATED CORRECTION FACTORS}

Pre-calculated Correction Factors, as a means of allowing coarse meshes to be used for the analysis of microstrips has been recently demonstrated[1] but so far they have not been applied to more complex structures. By including the PCFs in the analyses of the filter, which is done automatically by the enhanced FDTD computer program, the results for the three different meshes shown in Figure 7 are obtained. It can be seen that the results are all very similar regardless of the mesh size or position. The use of PCFs much reduces the sensitivity of the results on the choice of mesh and makes the choice of mesh a much simpler and less risky procedure as well as increasing overall accuracy for any given mesh.

A similar approach can be applied to the SSCN TLM method. In this case the fringing effects are accounted for by altering the characteristic impedance of the TLM lines which terminate at the edge of a strip. A mapping, analogous to the one described in [1] for FDTD can be readily derived and applied. Work is in progress to do this.

\section{CONCLUSION}

It has been shown that the predicted behaviour of a microstrip low pass filter is strongly dependent on both the size of the unit cell and the position of the strips with respect to the mesh for both the standard FDTD and the TLM methods. For the first time it has been shown that the use of Pre-calculated Correction Factors in FDTD for a complicated planar structure removes this strong dependence and allows a coarser mesh to be used for equivalent accuracy. Ongoing work is in progress to apply PCFs to the TLM SSCN algorithm with an expected similar improvement over the basic method.

\section{ACKNOWLEDGEMENT}

The author wishes to thank his colleagues, especially Dr. Dominique Paul and Dr. Ian Craddock for many helpful discussions.

\section{REFERENCE}

1 C. J. Railton, "The Inclusion of Fringing Capacitance and Inductance in FDTD for the Robust Accurate Treatment of Material Discontinuities“, IEEE Trans MTT-48, December 2000, pp. 2283-2288 




Figure 1 - A plan view of the low pass filter and the $0.5 \mathrm{~mm}$ mesh

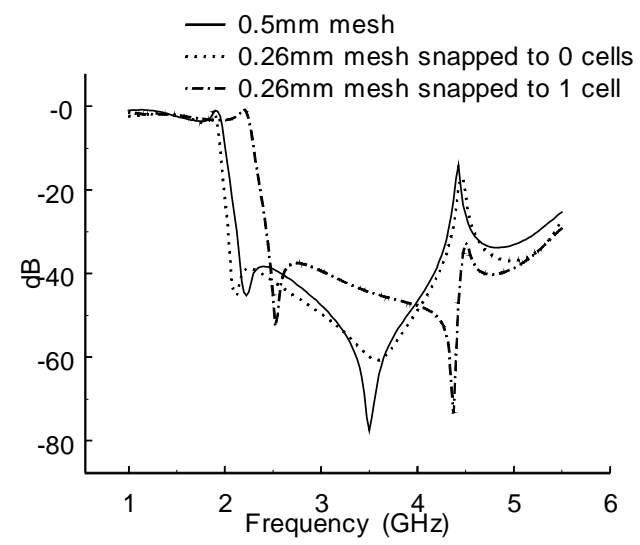

Figure 2 - Filter response for different FDTD meshes

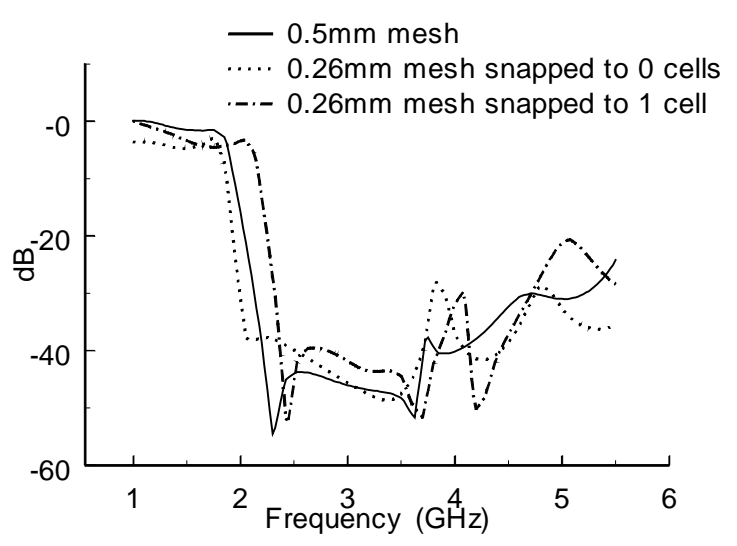

Figure 3 - Filter response for different TLM meshes 


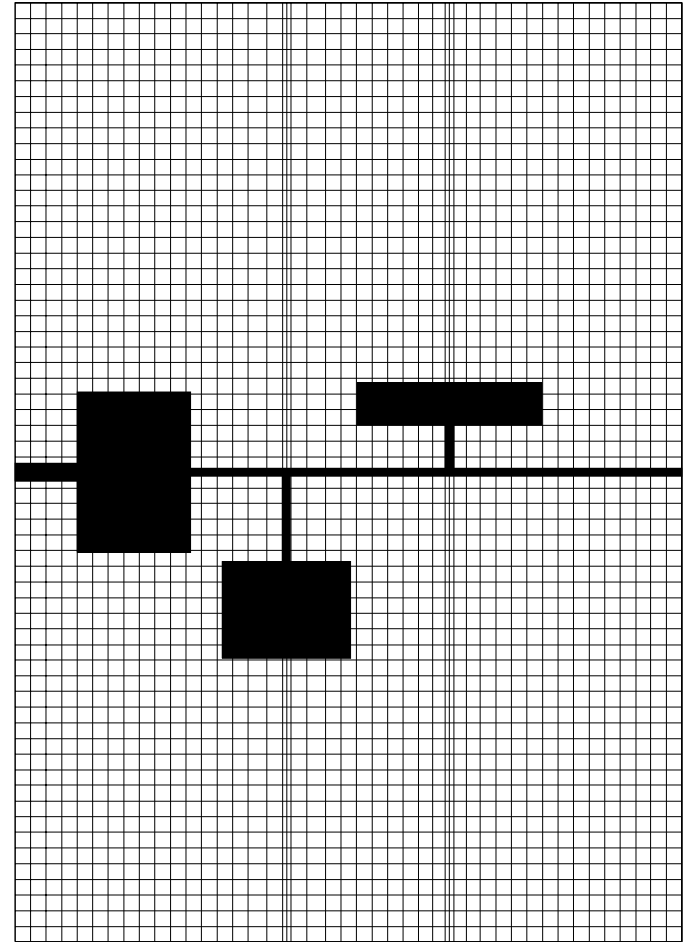

Figure 4 - The "f" mesh

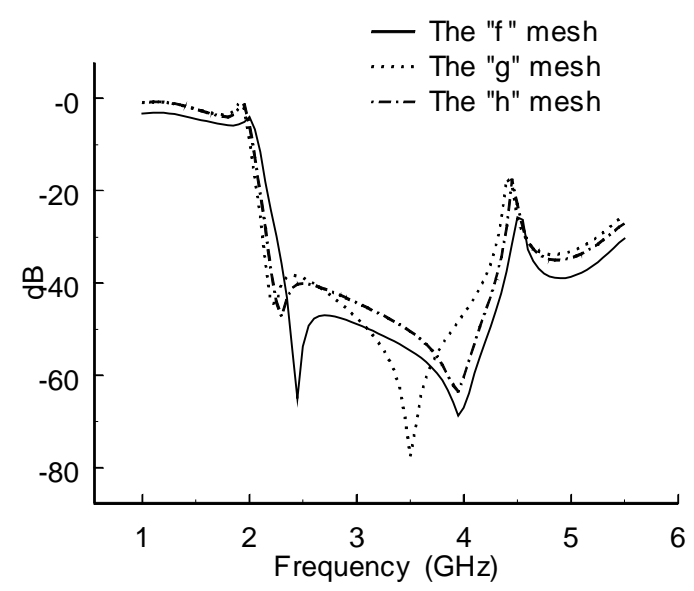

Figure 6 - Comparison of predicted results using FDTD with three similar non-uniform meshes

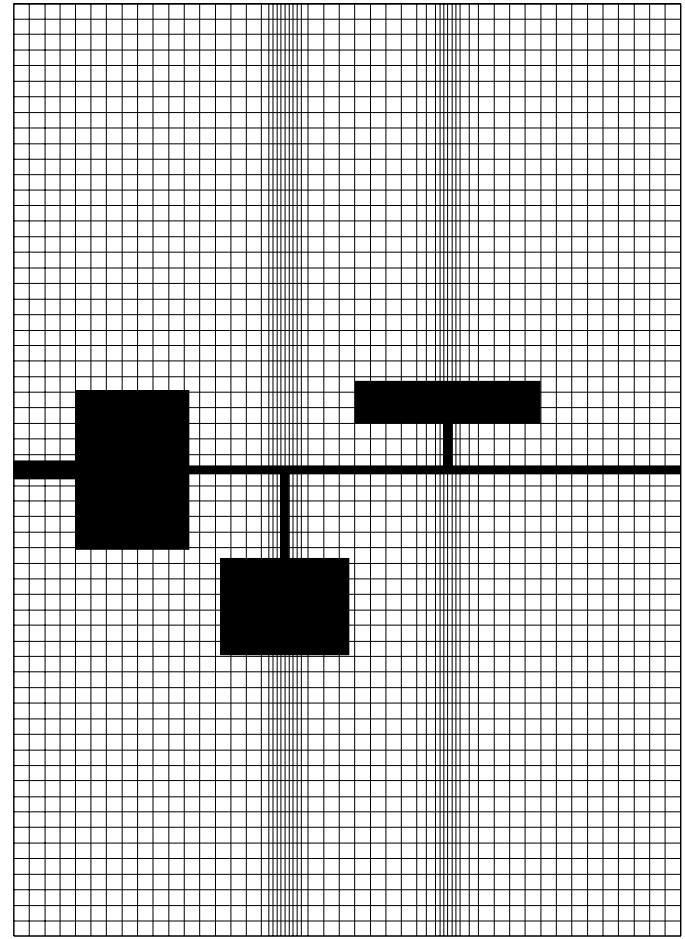

Figure 5 - The "h" mesh

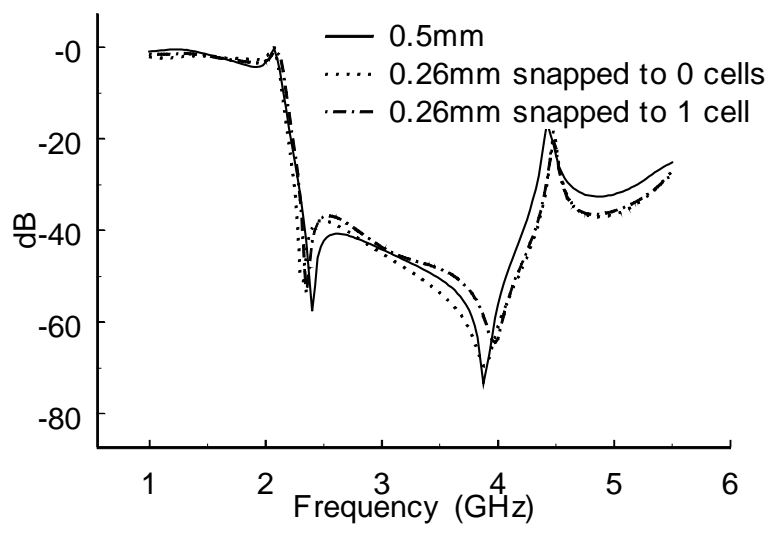

Figure 7 - Filter response for different FDTD meshes with PCFs 